$\frac{\text { MICHIGAN STATE }}{\text { U N I V E R S I T Y }}$

National Superconducting Cyclotron Laboratory

SPECTROSCOPY OF THE $2_{1}^{+}$STATE IN ${ }^{22}$ O AND SHELL STRUCTURE NEAR THE NEUTRON DRIP LINE

P.G. THIROLF, B.V. PRITYCHENKO, B.A. BROWN, P.D. COTTLE, M. CHROMIK, T. GLASMACHER, G. HACKMAN,

R.W. IBBOTSON, K.W. KEMPER, T. OTSUKA, L.A. RILEY, H. SCHEIT
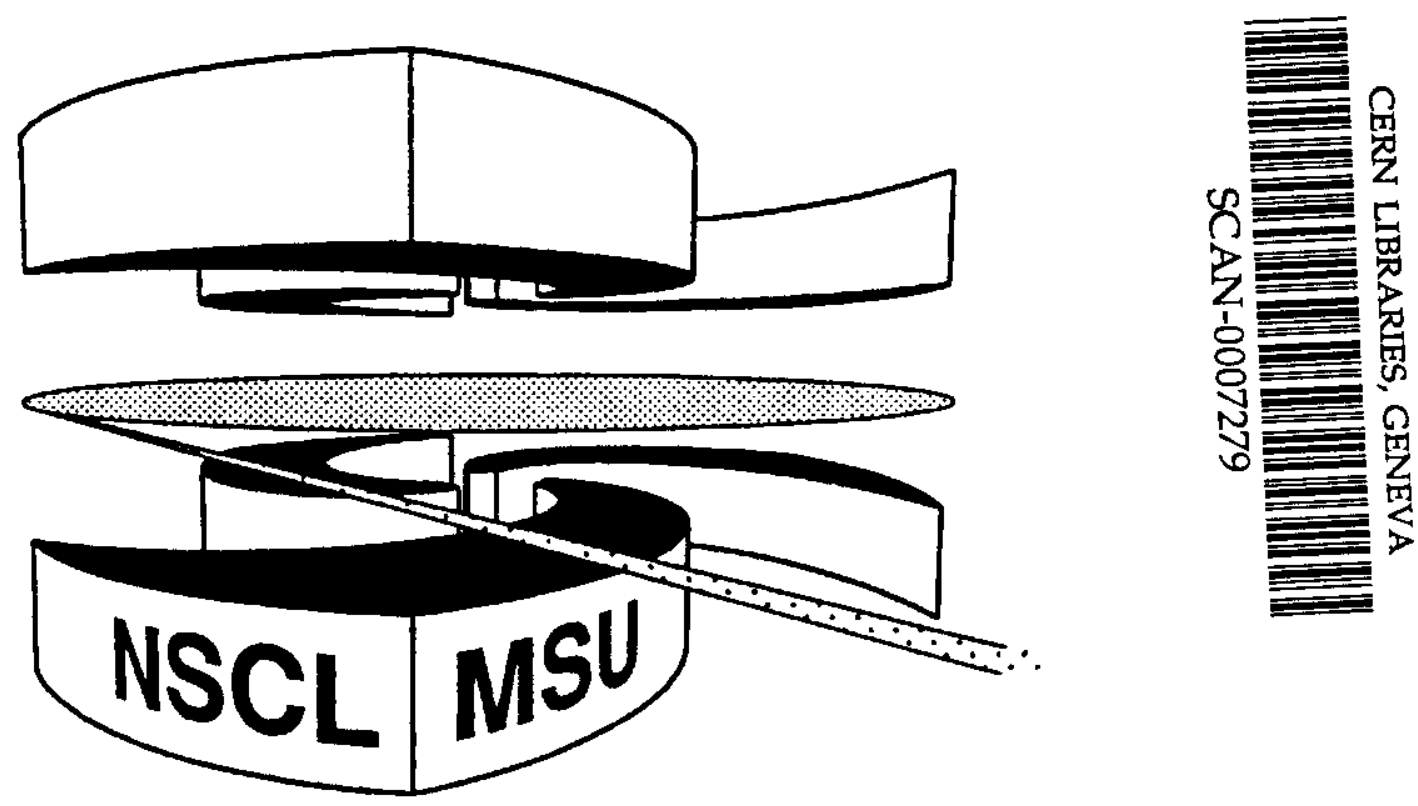

MSUCL-1153

MAY 2000 


\title{
Spectroscopy of the $2_{1}^{+}$state in ${ }^{22} \mathrm{O}$ and shell structure near the neutron drip line
}

P.G. Thirolf ${ }^{1}$, B. V. Pritychenko ${ }^{2,3}$, B.A. Brown ${ }^{2,3}$, P.D. Cottle ${ }^{4}$, M. Chromik ${ }^{1}$, T. Glasmacher $^{2,3}$, G. Hackman ${ }^{2 *}$, R.W. Ibbotson ${ }^{2 \dagger}$, K.W. Kemper ${ }^{4}$, T. Otsuka ${ }^{5,6}$, L.A. Riley ${ }^{7}$, H. Scheit ${ }^{2,3 \ddagger}$

${ }^{1}$ Ludwig Maximilians Universität München, D-85748 Garching, Germany

${ }^{2}$ National Superconducting Cyclotron Laboratory, Michigan State University, East Lansing, MI 48824, USA

${ }^{3}$ Department of Physics and Astronomy, Michigan State University, East Lansing, MI48824, USA

${ }^{4}$ Department of Physics, Florida State University, Tallahassee, FL 32306, USA

${ }^{5}$ Department of Physics, University of Tokyo, Hongo, Tokyo 113-0033, Japan

${ }^{6}$ RIKEN, Hirosawa, Wako-shi, Saitama 351-0198, Japan

${ }^{7}$ Department of Physics and Astronomy, Earlham College, Richmond, IN 47374, USA

(June 8, 2000)

\begin{abstract}
The energy and electromagnetic matrix element $B(E 2 \uparrow)$ for the $2_{1}^{+}$state
\end{abstract}

\footnotetext{
*Present address: Department of Physics and Astronomy, University of Kansas, Lawrence, KS 66045

${ }^{\dagger}$ Present address: Brookhaven National Laboratory, Upton, New York 11973

${ }^{\ddagger}$ Present address: Max-Planck-Institut für Kernphysik, Postfach 1039 80, D-69029 Heidelberg, Germany
} 
in ${ }^{22} \mathrm{O}$ have been determined via inelastic scattering of a beam of these radioactive nuclei from a ${ }^{197} \mathrm{Au}$ target. These results provide strong evidence for the existence of the $N=14$ subshell closure in ${ }^{22} \mathrm{O}$. This demonstrates that the shell structure in ${ }^{22} \mathrm{O}$ is similar to that of stable oxygen isotopes, even though this nucleus is only two neutrons away from the neutron drip line. These results are reproduced in the standard $s d$ shell model as well as with a an expanded space which also includes the $f_{7 / 2}$ and $p_{3 / 2}$ orbitals.

27.30.+t,25.70.De,23.20.Js,21.60.Cs 
The spin-orbit potential of a nucleon in the mean field of the nucleus is one of the central concepts in nuclear structure physics. The spin-orbit force was first invoked to explain the shell closures observed in stable and near-stable nuclei $[1,2]$, and the behavior of this interaction continues to be of great interest now. One of the major themes of nuclear structure studies with radioactive beams is to examine how changes in the spin-orbit potential near the drip lines affect shell structure [3-5]. In this letter, we report measurements of the energy and $B(E 2 \uparrow)$ electromagnetic matrix element of the $2_{1}^{+}$state in ${ }^{22} \mathrm{O}$ using the technique of intermediate energy heavy-ion inelastic scattering [6]. This nucleus is only two neutrons away from ${ }^{24} \mathrm{O}$, the heaviest particle-stable oxygen isotope $[7,8]$. The measurements reported here provide strong evidence for the existence of the $N=14$ subshell closure in ${ }^{22} \mathrm{O}$. A measurement of the energy of the $2_{1}^{+}$state in ${ }^{22} \mathrm{O}$ was reported earlier in a conference proceeding [9], but our measurement of the $B(E 2 \uparrow)$ provides important new evidence for the existence of the subshell closure. We demonstrate that the data reported here for ${ }^{22} \mathrm{O}$ can be understood with the standard $s d$ shell model [10] and with an expanded space [11] which also included the $f_{7 / 2}$ and $p_{3 / 2}$ orbitals. Both models use effective interactions derived from nuclei in and near the valley of stability.

The present experiment was performed at the National Superconducting Cyclotron Laboratory. The primary beam of $90 \mathrm{MeV} /$ nucleon ${ }^{40} \mathrm{Ar}$ was produced with the laboratory's K1200 cyclotron. Secondary beams were made via fragmentation of the primary beam in a $564 \mathrm{mg} / \mathrm{cm}^{2}{ }^{9} \mathrm{Be}$ production target located at the mid-acceptance target position of the A1200 fragment separator [12]. The ${ }^{22} \mathrm{O}$ secondary beam was produced with an energy of $55.6 \mathrm{MeV} /$ nucleon. Separation of beam isotopes was enhanced with a $130 \mathrm{mg} / \mathrm{cm}^{2}{ }^{27} \mathrm{Al}$ achromatic wedge placed at the second dispersive image of the A1200. The momentum acceptance of the A1200 was limited to $1.0 \%$ by slits located at the first dispersive image.

A $612 \mathrm{mg} / \mathrm{cm}^{2}{ }^{197} \mathrm{Au}$ foil was used as the secondary target. The secondary beam slowed down significantly in this target, and the mid-target beam energy was $50.6 \mathrm{MeV} / \mathrm{nucleon}$. The secondary beam was stopped in a cylindrical fast/slow plastic phoswich detector located at zero degrees. Both energy loss in the phoswich detector and time of flight relative to the 
cyclotron RF signal were used for particle identification. The zero degree detector subtended the scattering angles of $0^{\circ}$ to $3.50^{\circ}$ in the laboratory. The total integrated secondary beam measured by the zero degree detector was $2.92 \times 10^{7}$ particles.

The $\gamma$-rays were detected in an angular range of $56.5^{\circ}-123.5^{\circ}$ in the laboratory by an array of position sensitive $\mathrm{NaI}(\mathrm{Tl})$ detectors. A description of the array and details of the analysis of $\gamma$-ray spectra can be found in Ref. $[13,14]$. The $\gamma$-ray spectrum measured in coincidence with beam particles identified as ${ }^{22} \mathrm{O}$ in the zero degree detector appear in Fig. 1. In the top panel, the laboratory frame spectrum (uncorrected for the Doppler shift of the projectile) is shown. The $547 \mathrm{keV} 7 / 2^{+} \rightarrow 3 / 2_{g s}^{+} \gamma$-ray in the ${ }^{197} \mathrm{Au}$ target nucleus appears strongly in this spectrum. The projectile-frame (Doppler-corrected) spectrum is shown in the bottom panel of Fig. 1. A $\gamma$-ray appears at $3170 \pm 20 \mathrm{keV}$, which we assign to be the $2_{1}^{+} \rightarrow 0_{g s}^{+}$transition in ${ }^{22} \mathrm{O}$. This energy is consistent with that reported in recent conference proceedings by Azaiez and Belleguic et al. [9] from an in-beam fragmentation experiment experiment at GANIL. A second $\gamma$-ray seen at $1370 \mathrm{keV}$ in the GANIL experiment was not observed in our data. Since the reaction employed here preferably excites low-lying collective states and since multi-step excitations do not occur, the absence of the $1370 \mathrm{keV}$ photon in our data supports the suggestion by Azaiez and Belleguic et al. that this $\gamma$-ray might correspond to the $4^{+} \rightarrow 2^{+}$transition in ${ }^{22} \mathrm{O}$. The experiment reported here allows the measurement of a cross section (integrated over the projectile scattering angles $0^{\circ}$ to $3.50^{\circ}$ ) of $10.7 \pm 4.2 \mathrm{mb}$ for producing the $3170 \mathrm{keV} \gamma$-ray, assuming a $\gamma$-ray angular distribution corresponding to a pure E2 transition. Another $\gamma$-ray appears at $615 \pm 10 \mathrm{keV}$. We exclude the possibility that this $\gamma$-ray is the $2_{1}^{+} \rightarrow 0_{g s}^{+}$transition on the basis of systematics: the $2_{1}^{+}$ states of both ${ }^{18,20} \mathrm{O}$ are above $1.5 \mathrm{MeV}$. The $615 \mathrm{keV} \gamma$-ray may come from the decay of an excited state in ${ }^{21} \mathrm{O}$, which may be produced in a single neutron stripping reaction on the gold target. Such a neutron stripping reaction has been observed recently in a measurement of ${ }^{34} \mathrm{Si}$ (yielding the product ${ }^{33} \mathrm{Si}$ ) [15]. The $\gamma$-ray might connect a $615 \mathrm{keV}$ state in ${ }^{21} \mathrm{O}$ to the ground state, or it might connect two excited states in the same nucleus.

To analyze the cross section for the $3.17 \mathrm{MeV} \gamma$-ray while accounting for both the 
Coulomb and nuclear contributions to the reactions, we used the coupled channels code ECIS88 [16]. The analysis was performed assuming the mid-target beam energy of 50.6 $\mathrm{MeV} /$ nucleon. We did not have elastic scattering data for the present reaction, so we adopted the optical model parameters determined by Barrette et al. [17] for the scattering of ${ }^{17} \mathrm{O}$ from ${ }^{208} \mathrm{~Pb}$ at a laboratory energy of $84 \mathrm{MeV} /$ nucleon. To test the dependence of our results on the choice of optical model parameters, we have also employed an alternative set of optical model parameters by Mermaz et al. [25] derived from the scattering of ${ }^{16} \mathrm{O}$ from ${ }^{208} \mathrm{~Pb}$ at a laboratory energy of $49.5 \mathrm{MeV} /$ nucleon. The results are similar and are both discussed below. The standard vibrational form factor was used. Cross sections for multiple excitations in intermediate energy heavy-ion scattering are generally negligible [6], so we only considered single-step excitations here.

There are two coupling strengths (dynamic deformation parameters) involved in the ECIS calculations. The first, the "Coulomb deformation" $\beta_{C}$, reflects the deformation of the proton fluid in the nucleus and corresponds to the electromagnetic matrix element $B\left(E 2 ; 0_{g s}^{+} \rightarrow 2_{1}^{+}\right)$. The quantities $B\left(E 2 ; 0_{g s}^{+} \rightarrow 2_{1}^{+}\right)$and $\beta_{C}$ are related via the equation [18]

$$
\beta_{C}=\frac{4 \pi}{3 Z R_{0}^{2}}\left[B\left(E 2 ; 0_{g s}^{+} \rightarrow 2_{1}^{+}\right) / e^{2}\right]^{1 / 2}
$$

where the radius $R_{0}$ is given by $R_{0}=(1.20 \mathrm{fm}) A^{1 / 3}$.

The second deformation parameter in the calculation is the "nuclear deformation parameter" $\beta_{N}$. While the Coulomb deformation parameter is used to calculate the electromagnetic interaction between target and projectile, the nuclear deformation parameter is used in the nuclear potential to determine the matter interaction. The relationship between $\beta_{C}$ and $\beta_{N}$ depends on the proton and neutron contributions to the transition being studied and the sensitivity of the particular experimental probe used in the measurement to the proton and neutron contributions. The relationship between the proton and neutron contributions to the transition can be expressed as the ratio $M_{n} / M_{p}$ of the neutron and proton multipole matrix elements. The neutron (proton) multipole matrix element $M_{n(p)}$ is defined as

$$
M_{n(p)}=\left\langle J_{f}\left\|\Sigma_{n(p)} r_{i}^{\lambda} Y_{\lambda}\left(\Omega_{i}\right)\right\| J_{i}\right\rangle
$$


In the "standard" collective model where the neutron and proton oscillations are assumed to have the same amplitude, $M_{n} / M_{p}=N / Z$. Deviations from this value are systematically observed in semi-magic nuclei [19-21].

The sensitivity of the experimental probe $\mathrm{F}$ to neutron and proton contributions to the transition can be given as the ratio $b_{n}^{F} / b_{p}^{F}$, where $b_{n(p)}^{F}$ is the external field interaction strength of the probe $F$ with neutrons (protons) in the nucleus being studied. When $F$ is an electromagnetic probe, the ratio $b_{n}^{F} / b_{p}^{F}=0$, since the probe is sensitive only to the charge density and not to the neutron density. For low energy proton scattering $(50 \mathrm{MeV}$ and lower), $b_{n}^{F} / b_{p}^{F}=3$ and for low energy neutron scattering $b_{n}^{F} / b_{p}^{F}=1 / 3[19,20]$. To relate $\beta_{N}$ and $\beta_{C}$, we adopt the prescription of Ref. [20] in which the deformation length $\delta_{F}=\beta_{F} R$ for an experimental probe $F$ (where $R$ is the nuclear radius $R=r_{0} A^{1 / 3}$ ) is given by

$$
\frac{\delta_{F}}{\delta_{p}}=\frac{1+\left(b_{n}^{F} / b_{p}^{F}\right)\left(M_{n} / M_{p}\right)}{1+\left(b_{n}^{F} / b_{p}^{F}\right)(N / Z)} .
$$

Therefore, to fix the relationship between $\delta_{F}$ and $\delta_{p}$ (and therefore between $\beta_{N}$ and $\beta_{C}$ ) we must have values for $b_{n}^{F} / b_{p}^{F}$ and $M_{n} / M_{p}$. In the present experiment, the probe $F$ is ${ }^{197} \mathrm{Au}$, which contains both protons and neutrons, at $50 \mathrm{MeV} /$ nucleon. To extract $b_{n}^{F} / b_{p}^{F}$ for ${ }^{197} \mathrm{Au}$, we start from the assumption that

$$
b_{n(p)}^{F}=Z_{F} b_{n(p)}^{p}+N_{F} b_{n(p)}^{n}
$$

where $Z_{F}$ and $N_{F}$ are the proton and neutron numbers of the probe $F$, respectively. This assumption gives

$$
\frac{b_{n}^{F}}{b_{p}^{F}}=\frac{Z_{F} b_{n}^{p}+N_{F} b_{n}^{n}}{Z_{F} b_{p}^{p}+N_{F} b_{p}^{n}}
$$

which yields $b_{n}^{F} / b_{p}^{F}=0.820$ for ${ }^{197} \mathrm{Au}$ if we assume values of $b_{n}$ and $b_{p}$ which are valid at 50 $\mathrm{MeV}$.

We would need (and do not have) data on the $0_{g s}^{+} \rightarrow 2_{1}^{+}$transition in ${ }^{22} \mathrm{O}$ from a second experimental probe to determine $M_{n} / M_{p}$ (for examples of two cases where such data existed in the investigation of radioactive beams, see [22-24]). In the neighboring nucleus ${ }^{20} \mathrm{O}$, a 
comparison of measurements of the $0_{g s}^{+} \rightarrow 2_{1}^{+}$excitation using an electromagnetic probe and inelastic scattering of low energy protons in inverse kinematics [23] determined that $M_{n} / M_{p}=2.9 \pm 0.4$, which is considerably larger than $N / Z=1.5$. The ${ }^{20} \mathrm{O}$ result is typical of nuclei in which there is a closed proton shell and an open neutron shell since the valence neutrons play a large role in the excitation while the role of protons is limited to that given by the mechanism of core polarization. In the absence of data on ${ }^{22} \mathrm{O}$ from a second experimental probe, we adopt a value for $M_{n} / M_{p}(2.6)$ calculated using standard sd-shell calculations with the USD interaction [10].

The fit to the ${ }^{22} \mathrm{O}$ cross section data using ECIS yielded $\beta_{C}=0.21 \pm 0.04(B(E 2 \uparrow)=$ $21 \pm 8 \mathrm{e}^{2} \mathrm{fm}^{4}$ ) and $\beta_{N}=0.31 \pm 0.06$. The errors quoted are experimental errors and do not include a systematic error from the choice of $M_{n} / M_{p}$. Varying $M_{n} / M_{p}$ from the isoscalar limit of 1.5 to 3.0 changes the mean values of $\beta_{C}$ from 0.24 to 0.20 and $\beta_{N}$ from 0.25 to 0.32. To examine the dependence of our results on the optical model parameters, we also performed a fit with the parameter set obtained by Mermaz et al. [25]. This fit yielded a somewhat smaller value for $B(E 2 \uparrow)\left(15 \pm 7 e^{2} \mathrm{fm}^{4}\right)$ (for our choice of $M_{n} / M_{p}=2.6$ ). Varying the value for $M_{n} / M_{p}$ from the isoscalar limit of 1.5 to 3.0 , the parameter set by Mermaz et al. [25] yields deformation parameters $\left(\beta_{N}\right.$ and $\left.\beta_{C}\right)$ which are between $14 \%$ (for $M_{n} / M_{p}=1.5$ ) and $10 \%$ (for $M_{n} / M_{p}=3.0$ ) lower than the ones obtained with the optical model parameter set by Barrette et al. [17].

To check the reliability of our results, we extracted the cross section for the excitation of the $547 \mathrm{keV}$ state in ${ }^{197} \mathrm{Au}$ from the spectrum shown in the top panel of Figure 1, $15.8 \pm$ $4.0 \mathrm{mb}$. Using the same procedure as described above for ${ }^{22} \mathrm{O}$, we extract the electromagnetic matrix element $B(E 2 \uparrow)=4082 \pm 1012 e^{2} f m^{4}$, which agrees with the adopted value of $B(E 2 \uparrow)=4988 \pm 170 e^{2} \mathrm{fm}^{4}[26]$. This gives us confidence regarding the results of our measurement of ${ }^{22} \mathrm{O}$.

Figure 2 shows the data on the energies and $B(E 2 \uparrow)$ values for the $N>8$ even-even isotopes of oxygen (the data on ${ }^{16,18} \mathrm{O}$ are taken from Ref. [27]) as well as the results of shell model calculations which are described below. A comparison of the data on ${ }^{18} \mathrm{O}$ and ${ }^{20} \mathrm{O}$ 
demonstrates the importance of having data on both the energy and $B(E 2 \uparrow)$ value to judge the collectivity of the $2_{1}^{+}$state. The energy of the $2_{1}^{+}$state in ${ }^{20} \mathrm{O}$ is somewhat lower than in ${ }^{18} \mathrm{O}$, which might imply a greater degree of collectivity in ${ }^{20} \mathrm{O}$. However, the $B(E 2 \uparrow)$ value in ${ }^{20} \mathrm{O}$ is considerably lower, leading to the correct conclusion that the $2{ }_{1}^{+}$state in ${ }^{18} \mathrm{O}$ is more collective. In ${ }^{22} \mathrm{O}$, the energy of the $2_{1}^{+}$state is significantly higher than in ${ }^{20} \mathrm{O}$ and ${ }^{18} \mathrm{O}$, suggesting that the $N=14$ subshell gap that results from the filling of the $d_{5 / 2}$ neutron orbit is present in ${ }^{22} \mathrm{O}$. In this case, the $B(E 2 \uparrow)$ value for ${ }^{22} \mathrm{O}$ provides confirmation of the conclusion suggested by the $2_{1}^{+}$state energy, since it is is less than or equal to the $B(E 2 \uparrow)$ value in ${ }^{20} \mathrm{O}$. In short, the present results provide strong evidence for the existence of the $N=14$ subshell closure in ${ }^{22} \mathrm{O}$ even though this nucleus is only two neutrons away from the drip line nucleus ${ }^{24} \mathrm{O}$.

The existence of the $N=14$ subshell closure is in part a consequence of the ènergy splitting between the $d_{5 / 2}$ and $d_{3 / 2}$ spin-orbit partner orbits. Lalazissis et al. [5] calculated the spin-orbit splitting between these neutron orbits in the $\mathrm{Ne}$ and $\mathrm{Mg}$ isotopes (although not in the $\mathrm{O}$ isotopes) using relativistic mean field theory and concluded that the splitting is smaller near $N=30(\approx 5 \mathrm{MeV}$ for $\mathrm{Ne})$ than it is for the stable $\mathrm{Ne}$ isotopes $(\approx 7.5 \mathrm{MeV})$. However, these calculations predict a spin-orbit splitting at $N=14$ which is approximately equal to that for the stable isotopes. The existence of the subshell closure also depends on a separation between the energies of the $d_{5 / 2}$ and $s_{1 / 2}$ neutron orbits. In ${ }^{17} \mathrm{O}$ the $s_{1 / 2}$ level is only $0.87 \mathrm{MeV}$ above the $d_{5 / 2}$ levels, but by the time the $d_{5 / 2}$ orbit is mostly filled in ${ }^{22} \mathrm{O}$, the effective gap is widened to about $4 \mathrm{MeV}$ [28]. This change is built into the effective USD interaction via its influence on the masses and excitation energies in the more stable nuclei (in particular, the more stable nuclei with $Z>8$ and $N=14$ ) which were used to determine the empirical (USD) two-body matrix elements. This rapid change in the gap is not reproduced by HF calculations [29] in which closed-shell configurations are assumed. It has been suggested that this reflects the fact that neutron-rich nuclei such as ${ }^{22} \mathrm{O}$ and ${ }^{48} \mathrm{Ca}$ have better closed shells than the $N=Z$ nuclei ${ }^{16} \mathrm{O}$ and ${ }^{40} \mathrm{Ca} .[29]$.

It is reasonable to perform shell-model calculations for ${ }^{22} \mathrm{O}$ that use effective interactions 
derived from data on nuclei in and near the valley of stability which reproduce the mass dependence of the single-particle energies. We compare with the standard sd-shell calculations with the USD interaction [10] and expanded space calculations of $Z=8-14$ nuclei described by Utsono et al. [11] which incorporate the $f_{7 / 2}$ and $p_{3 / 2}$ orbitals in addition to the $s d$ shell with an interaction which starts with USD and then makes modifications to the monopole interaction. The USD calculations predict a $2^{+}$energy of $3.38 \mathrm{MeV}$ and a $B(E 2 \uparrow)$ value of $25 e^{2} \mathrm{fm}^{4}$ (with a standard neutron effective charge of $0.5[10]$ ), successfully reproducing our experimental results. The MCSM calculations give similar results of 3.13 $\mathrm{MeV}$ and $23 e^{2} \mathrm{fm}^{4}$, respectively, indicating that the pf-shell admixtures are small. The results of these calculations for ${ }^{18-24} \mathrm{O}$ are compared to the experimental data in Figure 2. The calculations for ${ }^{24} \mathrm{O}$ also predict a high $2_{1}^{+}$state energy and small $B(E 2 \uparrow)$ value. These results reflect the fact that $N=16$ also gives a subshell closure, this time from the filling of the $s_{1 / 2}$ neutron orbit. In these shell-model calculations only the neutron orbitals are active and the value for the $M_{p} / M_{n}$ ratio is 2.60 for all cases (with a neutron effective charge of 0.5 and a proton effective charge of 1.3 - see [30] for a discussion of how the effective charges enter into the $M_{p} / M_{n}$ ratio).

Khan and Van Giai [31] performed quasiparticle random phase approximation (QRPA) calculations of the $2_{1}^{+}$states of the neutron-rich oxygen isotopes with single particle energies and residual two-body interactions generated from Hartree-Fock calculations with a variety of nucleon-nucleon forces. While the QRPA calculations predict energies for the $2_{1}^{+}$states of ${ }^{18,20,22} \mathrm{O}$ which are approximately $2 \mathrm{MeV}$ higher than the experimental results, they provide fair agreement with the $B(E 2 \uparrow)$ data in ${ }^{20,22} \mathrm{O}$ (although they underpredict $B(E 2 \uparrow)$ in ${ }^{18} \mathrm{O}$ by almost $50 \%$ ). Khan and Van Giai also calculate $M_{n} / M_{p}$ values for these isotopes, and they successfully reproduce the experimental result for ${ }^{20} \mathrm{O}$. For ${ }^{22} \mathrm{O}$, the calculations predict $M_{n} / M_{p} \approx 3$, which is similar to the value for ${ }^{20} \mathrm{O}$. This theoretical result gives us additional confidence regarding our choice of $M_{n} / M_{p}=2.6$ for the analysis of the present data.

However, it is clear that an experimental determination of $M_{n} / M_{p}$ is critical for a thorough understanding of the structure of the low-lying states of ${ }^{22} \mathrm{O}$. In both ${ }^{18} \mathrm{Ne}$ and ${ }^{20} \mathrm{O}$, 
inverse kinematics proton scattering was used to provide the additional information needed to determine $M_{n} / M_{p}$ [22-24]. The new generation of radioactive beam facilities coming online shortly will be able to provide beams of ${ }^{22} \mathrm{O}$ intense enough to make proton scattering experiments feasible.

To summarize, we have reported measurements of the energy and $B(E 2 \uparrow)$ electromagnetic matrix element of the $2_{1}^{+}$state in ${ }^{22} \mathrm{O}$ using the technique of intermediate energy heavy-ion inelastic scattering. Our results provide strong evidence for the existence of the $N=14$ subshell closure in ${ }^{22} \mathrm{O}$. We have also demonstrated that the present data for ${ }^{22} \mathrm{O}$ can be understood using the standard $s d$ shell model with an effective interaction derived from nuclei in and near the valley of stability.

This work was supported by the National Science Foundation through grants PHY9605207, PHY-9528844 and PHY-9523974, and the State of Florida. 


\section{REFERENCES}

[1] O. Haxel, J.H.D. Jensen, and H.E. Suess, Phys. Rev. 75 (1949) 1766.

[2] M.G. Mayer, Phys. Rev. 75 (1949) 1969.

[3] J. Dobaczewski, I. Hamamoto, W. Nazarewicz, and J.A. Sheikh, Phys. Rev. Lett. 72 (1994) 981.

[4] M.M. Sharma, G.A. Lalazissis, W. Hillebrandt, and P. Ring, Phys. Rev. Lett. 72 (1994) 1431.

[5] G.A. Lalazissis, D. Vretenar, W. Pöschl, and P. Ring, Phys. Lett. B 418 (1998) 7.

[6] T. Glasmacher, Ann. Rev. Nucl. Part. Sci., 48 (1998) 1.

[7] D. Guillemaud-Mueller, J.C. Jacmart, E. Kashy et al. Phys. Rev. C 41 (1990) 937.

[8] M. Fauerbach, D.J. Morrissey, W. Benenson, B.A. Brown, M. Hellström, J.H. Kelley, R.A. Kryger, R. Pfaff, C.F. Powell, B.M. Sherrill, Phys. Rev. C 53 (1996) 647.

[9] F. Azaiez, Proc. Experimental Nuclear Physics in Europe (Seville, Spain), AIP conference proceedings 495 (1999) 171. F. Azaiez et al. Proc. Nuclear Structure 98 (Gatlinburg, TN, USA), AIP conference proceedings 48 (1998) 243. M. Belleguic et al. Physica Scripta (2000) in press.

[10] B.A. Brown and B.H. Wildenthal, Ann. Rev. Part. Nucl. Sci. 38 (1988) 29. The sd-shell energies obtained with the USD interaction are given on www.nscl.msu.edu/ brown/sde.htm.

[11] Y. Utsuno, T. Otsuka, T. Mizusaki, and M. Honma, Phys. Rev. C 60 (1999) 054315.

[12] B.M. Sherrill, D.J. Morrissey, J.A. Nolen Jr., and J.A. Winger, Nucl. Inst. and Meth. B 56 (1991) 1106.

[13] H. Scheit et al., Phys. Rev. Lett. 77 (1996) 3967. 
[14] H. Scheit, T. Glasmacher, R.W. Ibbotson, and P.G. Thirolf, Nucl. Inst. and Meth. A 422 (1999) 124.

[15] R.W. Ibbotson, T. Glasmacher, B.A. Brown, L. Chen, M.J. Chromik, P.D. Cottle, M. Fauerbach, K.W. Kemper, D.J. Morrissey, H. Scheit, and M. Thoennessen, Phys. Rev. Lett. 80 (1998) 2081.

[16] J. Raynal, Phys. Rev. C 23 (1981) 2571.

[17] J. Barrette, N. Alamanos, F. Auger, B. Fernandez, A. Gillibert, D.J. Horen, J.R. Beene, F.E. Bertrand, R.L. Auble, B.L. Burks, J.G. Del Campo, M.L. Halbert, R.O. Sayer, W. Mittig, Y. Schutz, B. Haas, and J.P. Vivien, Phys. Lett. B 209 (1988) 182.

[18] S. Raman, C.W. Nestor, Jr., and K.H. Bhatt, Phys. Rev. C 37 (1988) 805.

[19] A.M. Bernstein, V.R. Brown, V.A. Madsen, Phys. Lett. B 103 (1981) 255.

[20] A.M. Bernstein, V.R. Brown, V.A. Madsen, Comments Nucl. Part. Phys. 11 (1983) 203.

[21] M.A. Kennedy, P.D. Cottle and K.W. Kemper, Phys. Rev. C 46 (1992) 1811.

[22] L.A. Riley, J.K. Jewell, P.D. Cottle, T. Glasmacher, K.W. Kemper, N. Alamanos, Y. Blumenfeld, J.A. Carr, M. Chromik, S.E. Hirzebruch, R.W. Ibbotson, F. Maréchal, D.J. Morrissey, W.E. Ormand, F. Petrovich, H. Scheit, T. Suomijärvi, Phys. Rev. Lett. 82 (1999) 4196.

[23] J.K. Jewell, L.A. Riley, P.D. Cottle, K.W. Kemper, T. Glasmacher, R.W. Ibbotson, H. Scheit, M. Chromik, Y. Blumenfeld, S.E. Hirzebruch, F. Maréchal, and T. Suomijärvi, Phys. Lett. B 454 (1999) 181.

[24] L.A. Riley, P.D. Cottle, M. Fauerbach, T. Glasmacher, K.W. Kemper, B. V. Pritychenko, and H. Scheit (submitted to Phys. Rev. C).

[25] M.C.Mermaz, B.Berthier, J.Barrette, J.Gastebois, A.Gillibert, R.Lucas, J.Matuszek, A.Miczaika, E.Van Renterghem, T.Suomijarvi, A.Boucenna, D.Disdier, P.Gorodetzky, 
L.Kraus, I.Linck, B.Lott, V.Rauch, R.Rebmeister, F.Scheibling, N.Schulz, J.C.Sens, C.Grunberg, W.Mittig, Z.Phys. A 326 (1987) 353.

[26] C. Zhou, Nucl. Data Sheets 76 (1995) 399.

[27] S. Raman, C.H. Malarkey, W.T. Milner, C.W. Nestor, Jr., and P.H. Stelson, At. Data Nucl. Data Tables 36 (1987) 1.

[28] B. A. Brown, Rev. Mexicana de Fisica 39 Suplemento 2 (1993) 21.

[29] B. A. Brown, Phys. Rev. C 58 (1998) 220.

[30] B.A. Brown and B.H. Wildenthal, Phys. Rev. C 21 (1980) 2107.

[31] E. Khan and N. Van Giai, Phys. Lett. B 472 (2000) 253. 


\section{FIGURES}

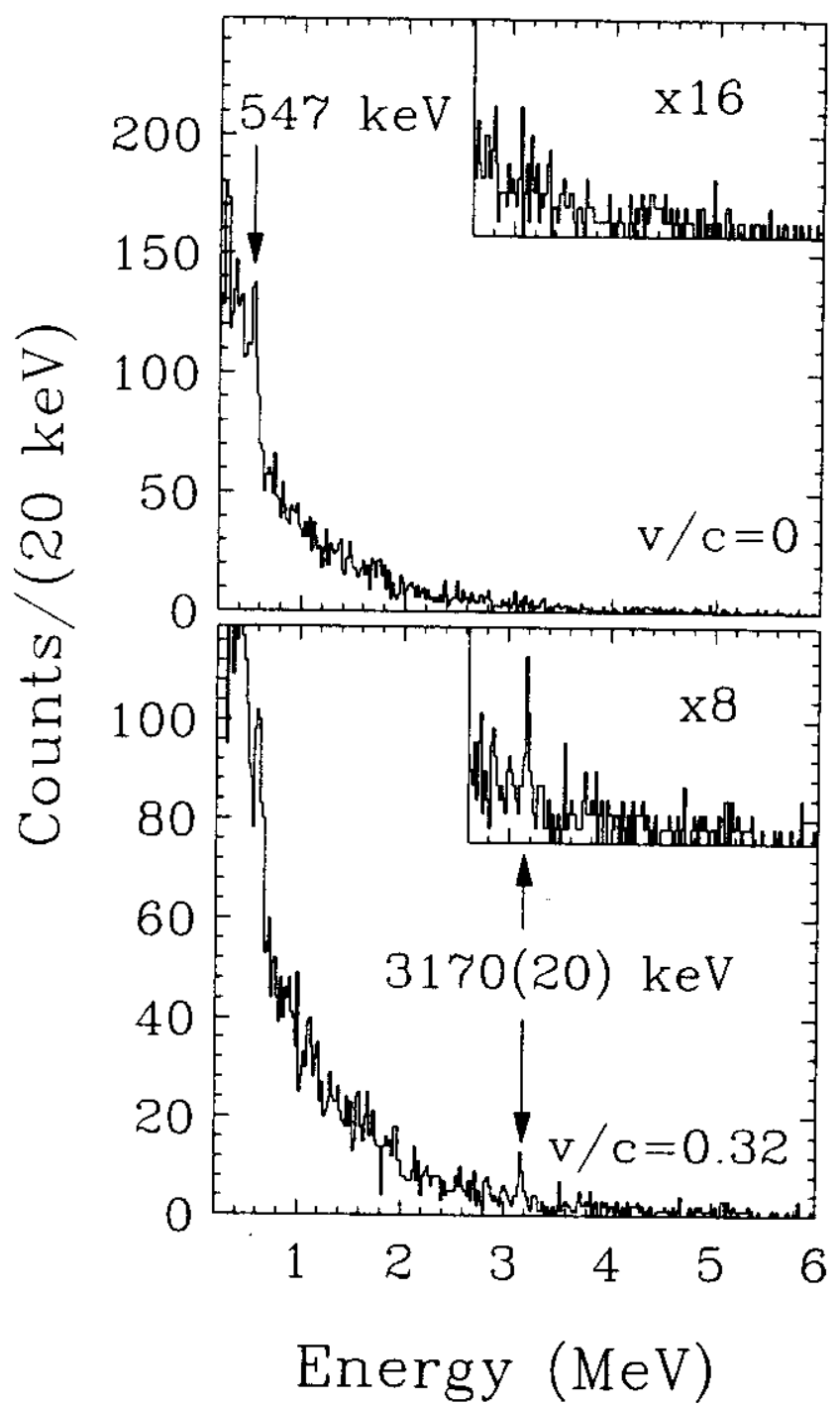

FIG. 1. In-beam photon spectra gated on ${ }^{22} \mathrm{O}$. The top panel shows the spectrum in the laboratory frame (without Doppler correction); the $7 / 2^{+} \rightarrow 3 / 2^{+}$transition in the gold target is visible as a peak. The bottom panel shows the projectile frame (Doppler-corrected) spectrum. The inserts show the same spectra above $2.6 \mathrm{MeV}$ on a different scale. 


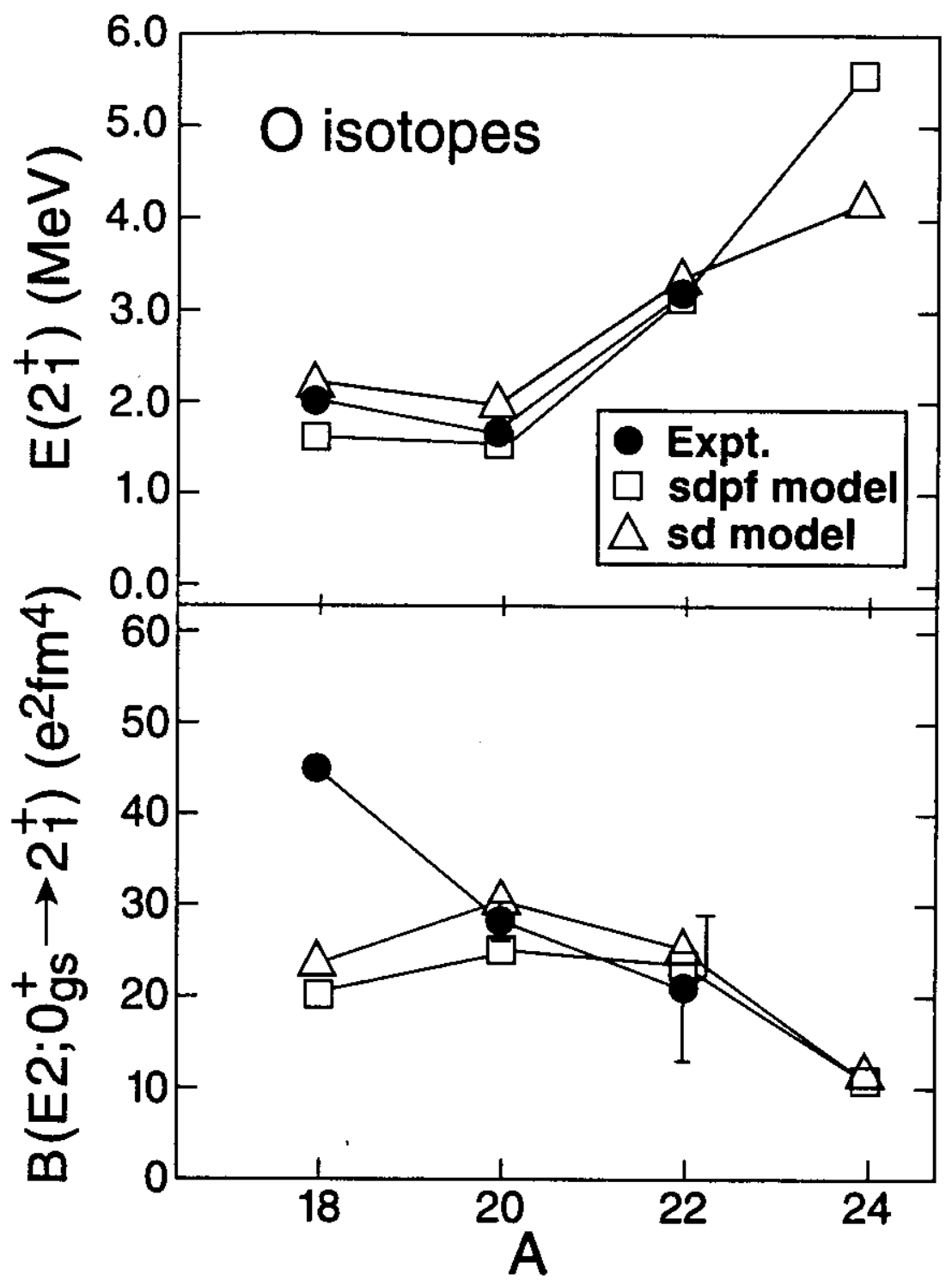

FIG. 2. The top panel shows the excitation energies of the $2_{1}^{+}$states in the $N>8$ even-even oxygen isotopes. The measured values (taken from [27] and the present work) are shown as solid shapes, and the calculated values for the standard $s d$-shell calculations [10] and the shell model calculations with an expanded space [11] are shown as open shapes. The bottom panel depicts the $B(E 2 \uparrow)$ values for the same nuclei. 